\title{
22nm FINFET Based High Gain Wide Band Differential Amplifier
}

\author{
Vasudeva $\mathrm{G}^{1}$ \\ Research Scholar, Electronics and Communication \\ Engineering Department, RV College of Engineering, \\ Bangalore-560059,Affliated to Visvesvaraya Technological \\ University,Belagavi,Karnataka,India \\ e-mail:devan.vasu921@gmail.com
}

\author{
Uma B V² \\ Professor and Dean Student Affairs, Electronics and \\ Communication Engineering Department, RV College of \\ Engineering, Bangalore-560059, Affilia ted to Visvesvaraya \\ Technological University, Bela gavi, Karnataka, India \\ e-mail: umabv@rvce.edu.in
}

Received: May 30, 2020. Revised: January 5, 2021. Accepted: January 26, 2021. Published: February 5, 2021.

\begin{abstract}
Differential Amplifier is a primary building block of analog and mixed signal circuit for pre-processing and signal conditioning of analog signal. FINFET devices with high-k gate oxide at $22 \mathrm{~nm}$ technology are predominantly used for high speed and low power complex VLSI circuits. FINFET based differential amplifiers are widely used in ADC's and signal Processing applications due to their advantages in terms of power dissipation. Analog front end of complex VLSI circuits need to offer high gain, higher stability and low noise figure. Designing of FINFET based VLSI sub-circuits requires proper design procedure that can provide designers flexibility in controlling the circuit performances. In this paper, differential amplifier is designed using model parameters of high-k FINFET in $22 \mathrm{~nm}$ technology. The conventional procedures for designing MOSFET based differential amplifier are modified for designing FINFET based differential amplifier. Schematic capture is carried out in Cadence environment and simulations are obtained considering $22 \mathrm{~nm}$ FINFET PDK. The performance metrics are evaluated and optimized considering multiple iterations. The designed differential amplifier has slew rate of $6 \mathrm{~V} / \mu \mathrm{Sec}$ and settling time of $0.9 \mu \mathrm{Sec}$ which is a desired metric for ADCs. Power Supply Rejection Ratio (PSRR) is $83 \mathrm{~dB}$ and dynamic range is $1.6754 \mathrm{~V}$. Open loop DC gain of DA is achieved to be $103 \mathrm{~dB}$ with phase margin of $63^{\circ}$ that demonstrates the advantages of $\mathrm{DA}$ designed in this work suitable for analog front end.
\end{abstract}

Key Words - FINFET, Differential Amplifier (DA), CMRR, PSRR, High Gain, Wide Band

\section{I.INTRODUCTION}

Differential Amplifier(DA) is a fundamental building block of complex analog circuits. The DA circuits processes the input signal and amplifies the signal with high gain minimizing noise at the output. The operational amplifier module has the DA in the front end for this purpose. CMOS circuits have been predominantly used in design of complex analog circuits and in the last few years new devices such as FINFETs are used in place of MOS transistors. In the year 1987 Balestra et al. [1] proposed the Double Gate MOSFET (DG-MOSFET) that had advantages over MOSFET in terms of increased current and reduced short-channel effects. Using vertical silicon layer called as FIN with two lateral gates gave rise to one of the best transistor structures. FINFET based amplifier performs better when compared with MOSFET based amplifiers. Further tothis another gate at the top resulted in trigate transistors with two lateral channels. It is also required to design circuits using FINFETs and hence it is required to understand the voltage and current relationship in FINFETs. There are two types of operational amplifiers such as two stage and folded cascode amplifiers. The two-stage amplifier comprises of differential amplifier and source follower or second stage amplifier circuit. For design of high gain operational amplifier with wide band width support the differential amplifier (DA) need to be designed appropriately setting standard design procedures for the transistor geometries. Design MOSFET based DA are based on well-known conventional methods, it is required to design DA based on FINFETs. In this paper, a detailed discussion on design of DA is presented considering FINFET VI characteristics. FINFET is one of the recent technologies used for low voltage analog and digital applications. Different features of FINFET created single stage Differential amplifier compared with Conventional CMOS Single-stage Differential amplifier. FINFET circuit offers best performance than the MOSFET and CNFET at the cost of power consumption. The low leakage, low power dissipation and high current driving capabilities of the FINFET are the basic building blocks of Differential amplifier. From the recent studies of Differential amplifier design using FINFET, Amir Baghi Rahin et al. [2] proposed a double gate transistor with independent gate using FINFET and simulated the same using a supply voltage of $1 \mathrm{~V}$. In the design a compensation capacitor of value $2.2 \mathrm{PF}$ was used. Vikhe et al. [3] in their work designed differential amplifier using FINFET and evaluated itsperformances. Power consumption of $58 \mu \mathrm{W}$ was observed in their work on FINFET based DA at $1 \mathrm{~V}$ power supply. DC gain of $52 \mathrm{~dB}$ with unity gain frequency of $6.4 \mathrm{MHz}$ and phase margin of $71^{\circ}$ were obtained. The DA circuit designed is having limited operating frequency for high speed applications. S. Hsieh et al. [4] discuss design of 12-bit SAR ADC using adaptive time domain comparator and, they incorporated noise optimization method. The DA circuit designed has gain of less than $50 \mathrm{~dB}$. Y. Chen et al. [5] designed a 9-bit, $1.46 \mathrm{~mW}$ SAR ADC using $65 \mathrm{~nm}$ technology. They used differential amplifier in their design to achieve an amplified output at the output stage. M. Yoshika et al. [6] proposed a 10-bit, $820 \mu \mathrm{W}$ SAR ADC with on-chip digital calibration method, the high power dissipation in the ADC is 
contributed by power dissipation in DA circuit. H. Choi et al. [7] in their work used mid-code calibration technique for SHA based free dual channel nyquist ADC. DA circuit in their work is found to operate in $\mathrm{MHz}$ frequency range and is limited for high speed ADCs. Tai-ji et al. [8] designed a CMOS SAR ADC using asynchronous pipelined architecture and achieved a highpower dissipation $3.5 \mathrm{~mW}$ using $1.0 \mathrm{~V}$ supply voltage. DNL and INL achieved are $0.71 \mathrm{LSB}$ and $0.70 \mathrm{LSB}$, respectively. Murmann [9] worked on transistor scaling and ADC architecture design using different sub circuits. Most of the DA circuits have been designed to achieve high gain but have limited operating frequency of less than $60 \mathrm{MHz}$. FINFET based DA circuit have demonstrated higher operating frequencies in $\mathrm{ADC}$ circuit design [10] and discussions on transistor scaling and its limitations are presented in [11]. Next section discusses the concept of FINFET and design procedure for DA circuit using FINFETs.

\section{FINFET}

Chenming HU, Tsu-Jae King-Liu and Jeffrey Bokor from University of California have developed the FINFET with Double Gate (DG) [13] to control the flow of current in the channel. The gate to channel coupling is improved due to double gates and with control mechanism on both sized channel size is reduced [13]. DG-FETs a re operated at lower threshold volta ges and reduce power dissipation [12]. Figure 1 shows the structure of FINFET with double gate (top and bottom) [14] placed on the insulator, the source and drain regions are conned through the channel. The circuit topology or the small signal model of theFINFET is shown in Figure 2. The intrinsic circuit comprises of parasitic capacitances $\mathrm{C}_{\mathrm{gd}}, \mathrm{C}_{\mathrm{gs}}$ and $\mathrm{C}_{\mathrm{ds}}$ a long with the parasitic resistance $\mathrm{R}_{\mathrm{gd}}, \mathrm{R}_{\mathrm{gs}}, \mathrm{R}_{\mathrm{ds}}$ and $\mathrm{R}_{\text {sub. }}$. The capacitances $\mathrm{C}_{\mathrm{pg}}$ and $\mathrm{C}_{\mathrm{pd}}$ are considered at low frequencies with pinch-off condition. The parameters Lg, $\mathrm{Rg}$, Rs and $\mathrm{Rd}$ are computed considering Vgs above pinch-off.

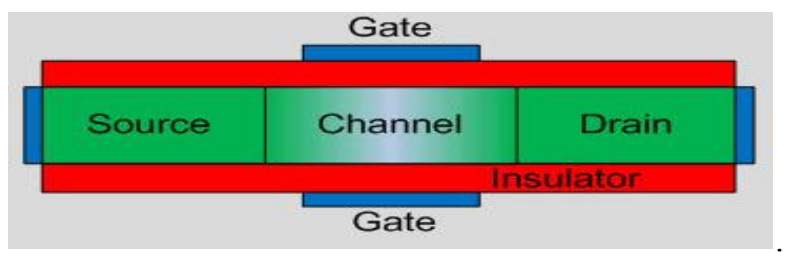

Fig. 1 - Structure of FINFET(Double ga te) [15]

The small signal model and model file of FINFET considered from Predictive Technology Model (PTM) is considered for design of ADC.The device parameters a re presented in Table 1. The source doping and drain doping concentration based on Gaussian doping is considered at $1 \mathrm{e}+19 / \mathrm{cm}^{-3}$, the dielectric constant of channel is 11.7 and the dielectric constant of insulator is 3.9.

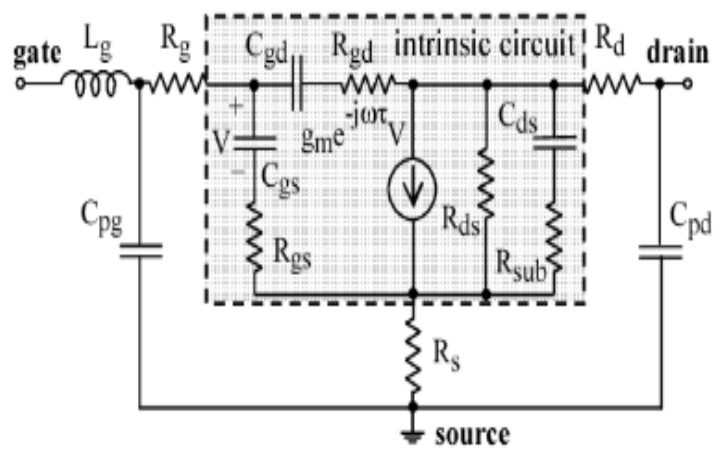

Fig. 2-Sma ll signal equivalent circuit for FINFET [1 6]

The bandgap and affinity of channel material is considered at $1.12 \mathrm{eV}$ and $4.05 \mathrm{eV}$ with gate contact work function of $4.6 \mathrm{eV}$. Mobility of electrons and saturation velocity is considered at $1400 \mathrm{~cm}^{2} / \mathrm{Vs}$ and $1.07 \mathrm{e}+07 \mathrm{~cm} / \mathrm{s}$. Considering these structural and electrical properties for FINFET the device model is simula ted for its input and output characteristics.

Table 1-FINFET Device Parameters

\begin{tabular}{|l|l|l|}
\hline Parameter & $\begin{array}{l}\text { Value (This } \\
\text { Work) }\end{array}$ & Value [12] \\
\hline Channel length & $22 \mathrm{~nm}$ & $32 \mathrm{~nm}$ \\
\hline Oxide thickness 1 & $2.5 \mathrm{~nm}$ & $1.6 \mathrm{~nm}$ \\
\hline Oxide thickness 2 & $2.5 \mathrm{~nm}$ & $1.6 \mathrm{~nm}$ \\
\hline Gate length & $22 \mathrm{~nm}$ & - \\
\hline $\begin{array}{l}\text { Source/drain } \\
\text { extensionlength }\end{array}$ & $50 \mathrm{~nm}$ & $32 \mathrm{~nm}$ \\
\hline $\begin{array}{l}\text { Gate to source/drain } \\
\text { overlap }\end{array}$ & $2 \mathrm{~nm}$ & - \\
\hline Work function & $4.6 \mathrm{eV}$ & $4.5 \mathrm{eV}^{20} \mathrm{~cm}^{-3}$ \\
\hline Source/Drain doping & $1 \times 10^{19} \mathrm{~cm}^{-3}$ & $2 \times 10^{-3}$ \\
\hline
\end{tabular}

Figure 3(a) and 3(b) presents the input and output characteristics for the FINFET considered with the structural parameters as in Table 1. The parameters chosen in this work is compared with the parameters that have been considered in [12]. The technology selected in this work is $22 \mathrm{~nm}$. The input is obtained by setting the drain voltage at $0.5 \mathrm{~V}$ and $1 \mathrm{~V}$. The output characteristic is obtained by setting the gate voltage between 0 $\mathrm{V}$ to $1 \mathrm{~V}$ with incremental step so $0.1 \mathrm{~V}$. 


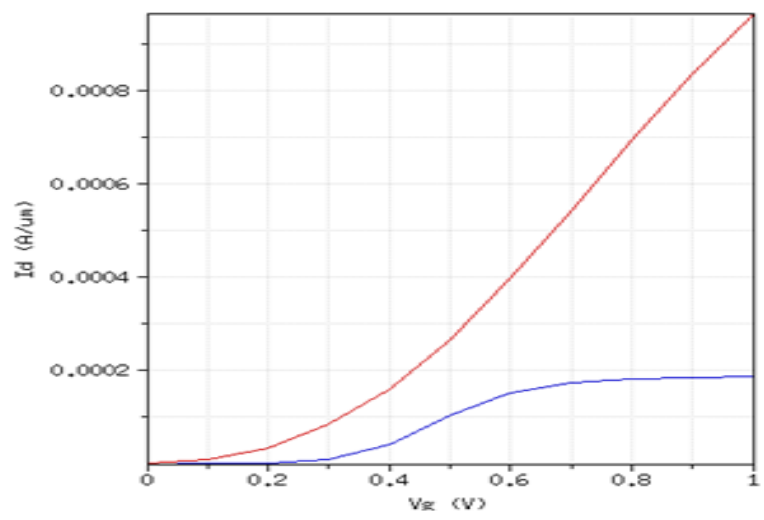

Fig 3(a)- Input Characteristics

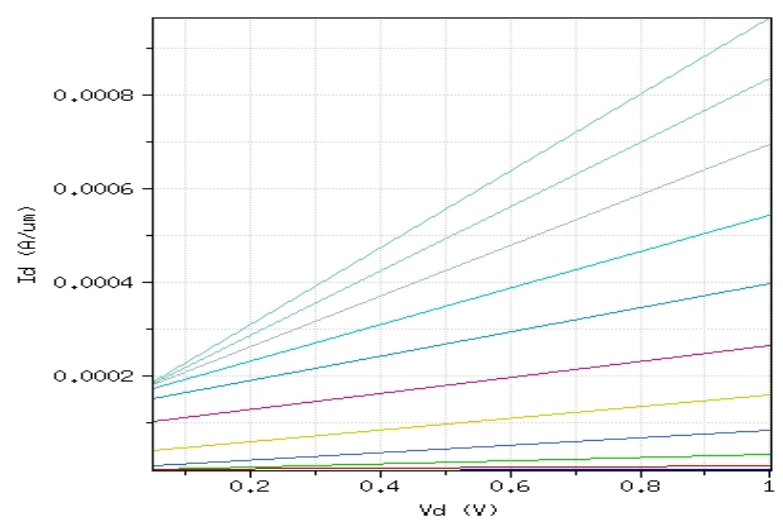

Fig 3(b)-Output characteristics

Figure 4(a) presents the power dissipation and transfer characteristics of inverter based on FINFET device. The maximum power dissipation is observed to be less than $800 \mathrm{nW}$ and transition width is less than $0.12 \mathrm{~V}$.

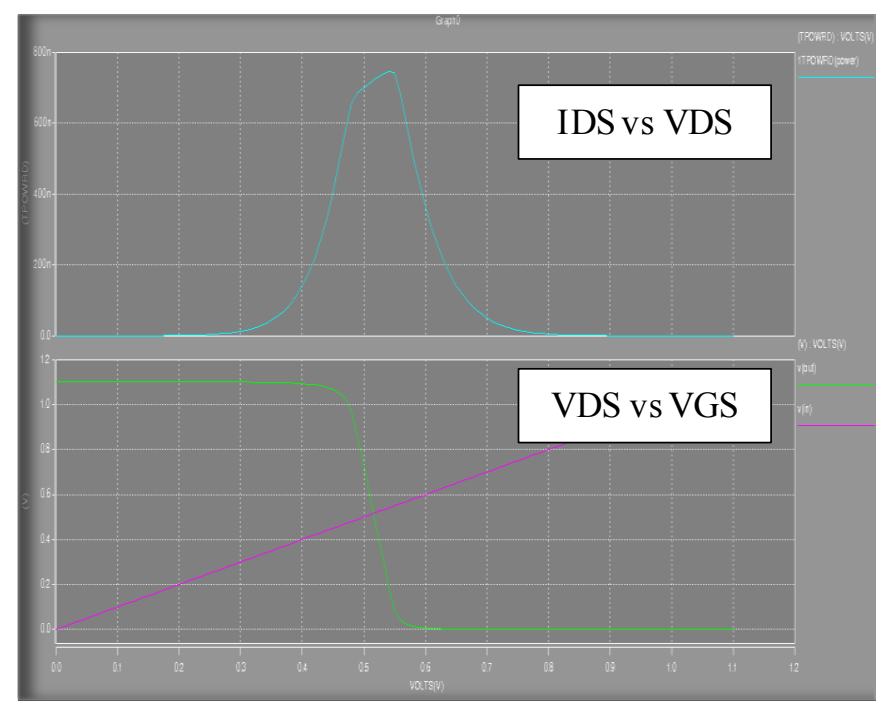

Fig. 4(a)-Power dissipation and transfer Characteristics of Inverter

Figure 4(b) presents the leakage current analysis for FINFET based inverter circuit. The leakage current during positive switching and negative switching current is observed to be less than $9 \mu \mathrm{A}$.

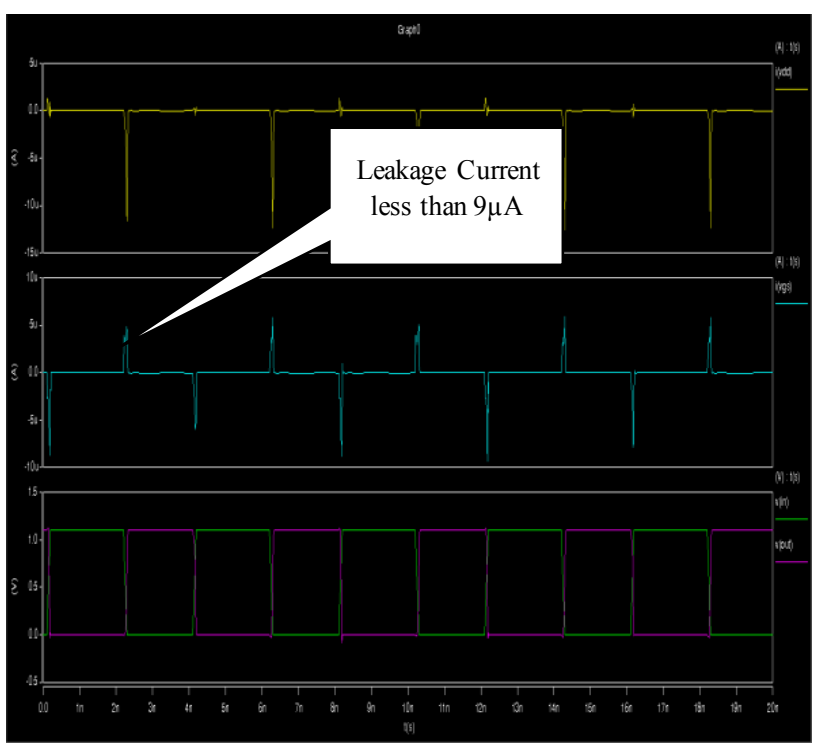

Fig 4(b)-Lea kage Current analysis of Inverter characteristics for 22nm high-k FINFET

\section{III.DIFFERENTIAL AMPLIFIER DESIGN}

The Differential Amplifier (DA) at the input stage of the OPAMP a chieves larger ga in lea ding to improvement in noise performance and offset. Figure 5 presents the circuit schematic of differential amplifier using FINFET. The two gates of FINFET a re shorted and are used as single gate transistor device. Transistors M1 and M2 are the common source amplifiers with P-channel FINFET (M3 and M4) used as current mirrors. Transistor M5 is the bias transistor that is required to set the maximum current that can flow in the two amplifiers. Transistor M8 is the N-channel FINFET current mirror used as current reference providing bias current for transistor M5. The load capacitance CL is set across which the output voltage is observed.

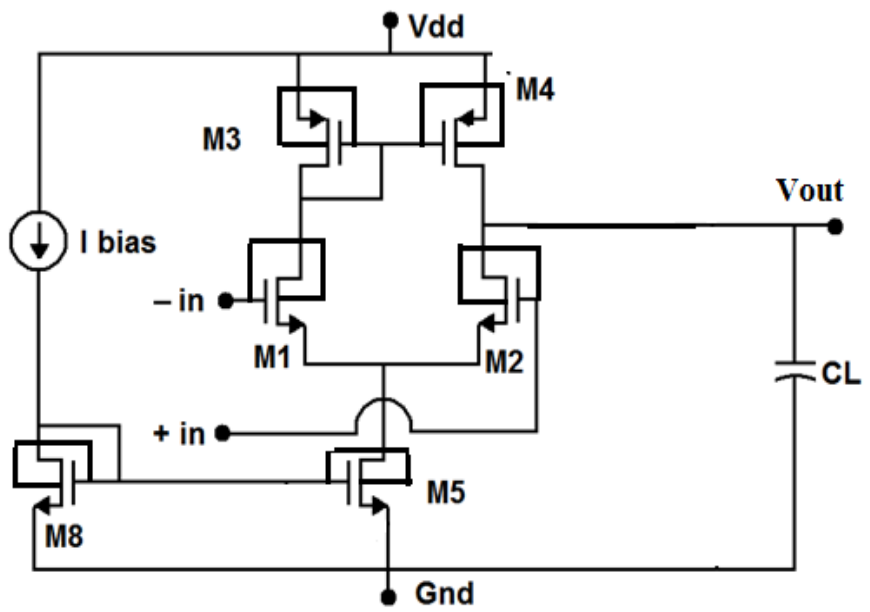

Fig. 5-FINFET basedDA

In typical $\mathrm{A} / \mathrm{D}$ converter applications, the $\mathrm{DA}$ which is a so used as comparator must slew its output quickly and without oscilla tion once the input thresholds a re crossed. The slew rate for the DA or comparator is measured by giving a step input 
signal and measuring the time it takes for the DA to reach the final output value. The circuit schematic for the DA is captured in Cadence environment. The transistor geometries for the M1 and $\mathrm{M} 2$ are set to $100 \mathrm{~nm}$ and the transistor geometries for $\mathrm{M} 3$ and $\mathrm{M} 4$ a re set to $200 \mathrm{~nm}$, transistor geometries for M5 and M8 are set to $400 \mathrm{~nm}$ supporting maximum driving current of 100 $\mu \mathrm{A}$.

\section{IV.DESIGN OF DIFFERENTIAL AMPLIFIER TRANSISTOR GEOMETRIES}

Table 2 presents the DA design specifications considered for the requirement of OPAMP design [11]. The slew rate is assumed to be greater than $6 \mathrm{~V} / \mu \mathrm{S}$ and the power dissipation is set to be less than $50 \mu \mathrm{W}$ as per the design specifications of high speed ADCs. The factors $\mathrm{K}_{\mathrm{p}}{ }_{\mathrm{p}}$ and $\mathrm{K}_{\mathrm{n}}{ }_{\mathrm{n}}$ a re computed considering the mobility and parasitic capacitance parameters obtainedfrom the model file.

Table 2-DA Design Specifications

\begin{tabular}{|l|l|}
\hline Parameters & Value \\
\hline Slew Rate $=$ SR & $>6 \mathrm{~V} / \mu \mathrm{S}$ \\
\hline $\mathrm{V}_{\text {out }}$ range & $= \pm 2 \mathrm{~V}$ \\
\hline ICMR & $=0.15$ to $0.8 \mathrm{~V}$ \\
\hline Phase margin & $60^{0}$ to $75^{\circ}$ \\
\hline Open loop ga in & $>100 \mathrm{~dB}$ \\
\hline Power Dissipation & $\leq 50 \mu \mathrm{W}$ \\
\hline $\mathrm{V}_{\text {th }}=\left|\mathrm{V}_{\text {tp }}\right|$ & $=0.25 \mathrm{~V}$ to $0.45 \mathrm{~V}$ \\
\hline $\mathrm{K}_{\mathrm{p}}^{\prime}=\mu_{\mathrm{p}} \mathrm{C}_{\text {ox }} / 2$ & $=-455 \mu \mathrm{A} / \mathrm{V}^{2}$ \\
\hline $\mathrm{K}_{\mathrm{n}}^{\prime}=\mu_{\mathrm{n}} \mathrm{C}_{\mathrm{ox}} / 2$ & $=1085 \mu \mathrm{A} / \mathrm{V}^{2}$ \\
\hline
\end{tabular}

Design procedure for MOSFET basedDA is discussed in detail by Allen Hollberg [17]. In this work the design procedure is fine tuned to compute the transistor geometries of FINFETs for DA circuit schematic. Table 3 summarizes the design procedure for FINFET based DA Design.

Table 3-Transistor geometry

\begin{tabular}{|c|c|c|}
\hline $\begin{array}{c}\text { Transistor } \\
\text { Number }\end{array}$ & $\begin{array}{c}\text { W } \\
\text { (MOSFET) } \\
\mathbf{n m}\end{array}$ & $\begin{array}{c}\text { W } \\
\text { (FINFET) } \\
\mathbf{n m}\end{array}$ \\
\hline M1 & 200 & 100 \\
\hline M2 & 200 & 100 \\
\hline M3 & 1200 & 600 \\
\hline M4 & 1200 & 600 \\
\hline M5 & 800 & 400 \\
\hline M8 & 1600 & 800 \\
\hline
\end{tabular}

Based on the identified transistor widths the schematic capture is modeled in Cadence and simulation results are obtained to identify the performances of DA a mplifier.

\section{RESULTS ANDDISCUSSIONS}

The performance parameters such as DC ga in, open loop gain, output swing, CMRR, PSRR and transconductance are measured considering standard test models. The results obtained a re discussed in detail for the DA circuits designed using FINFET. The DC gain of the DA is measured considering the test configuration shown in Figure 6 which provides provision for direct measurement of $\mathrm{v}_{\mathrm{o}} / \mathrm{v}_{\text {in }}$ at DC. A load capacitance of CL is included at the output side and the noninverting input is swept with AC signal with $\mathrm{V}_{\mathrm{O}}$ (offset voltage) of $0.5 \mathrm{mV}$. Adjusting DC gain will help in improvising all other parameters. Variation in load ca pacitance impacts the wide band operation of DA circuit.

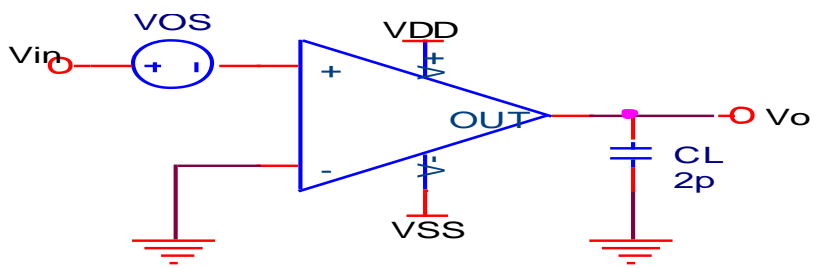

Figure 6-Configurations to find DC Ga in

From the simula tion results for measuring DC Ga in as shown in Figure 7, the maximum ga in is found to be $83 \mathrm{~dB}$ and the phase margin is $65^{\circ}$ measured at $3 \mathrm{GHz}$ operating frequency.

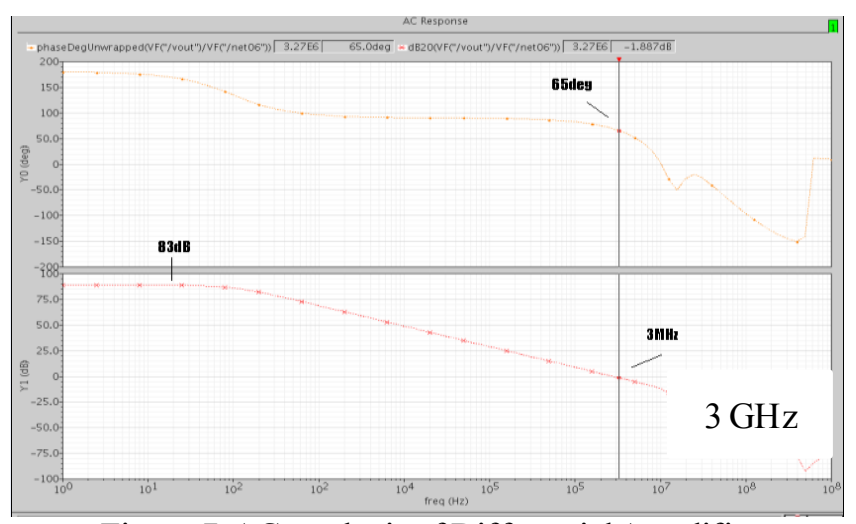

Figure 7-AC a nalysis of Differential Amplifier

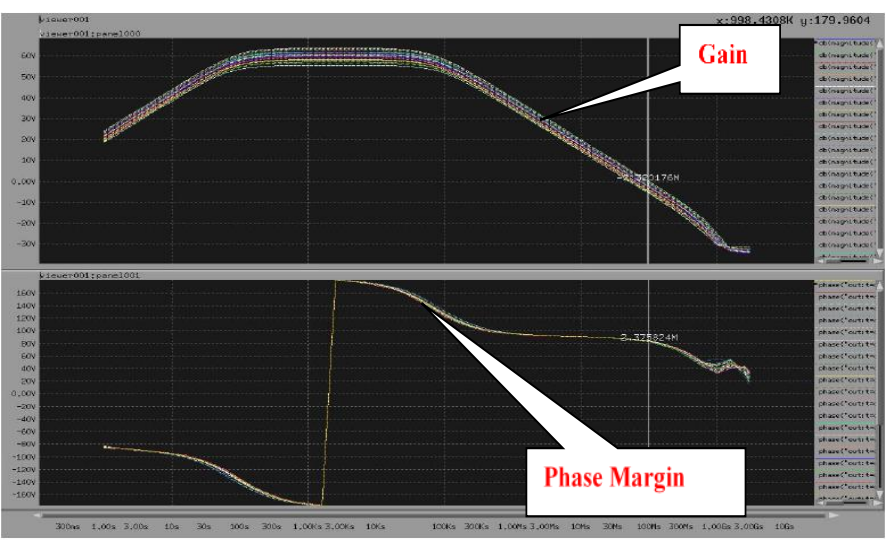

Fig. 8-Gain and phase-margin plots ofDA with varying $\mathrm{W}_{\text {eff }}$ 
The expected gain of Differential amplifier is a round 100 to 120 $\mathrm{dB}$. The achieved gain is a round $107 \mathrm{~dB}$. The phase noise margin increases the stability of the Differential amplifier. The desired pha se noise margin is a round $55^{\circ}$ to $75^{\circ}$ and the a chieved Phase noise margin is $63^{\circ}$.In order to measure the output voltage swing the test configuration in Figure 9 is considered. The input is applied at the inverting terminal with input resistance of $1 \mathrm{M}$ $\mathrm{Ohm}$ and a feedback is introduced with resistance of $10 \mathrm{MOhm}$. The inverting configuration is measured for its output voltage swing by setting the inputs to various voltage levels. The output is measured for its voltage swing and the test results a re shown in Figure 10.

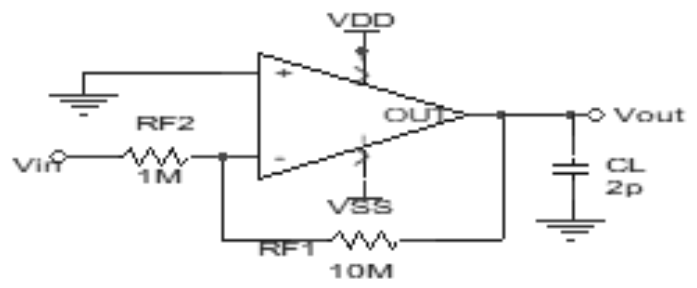

Figure 9- Configuration for the measurement of the output swing

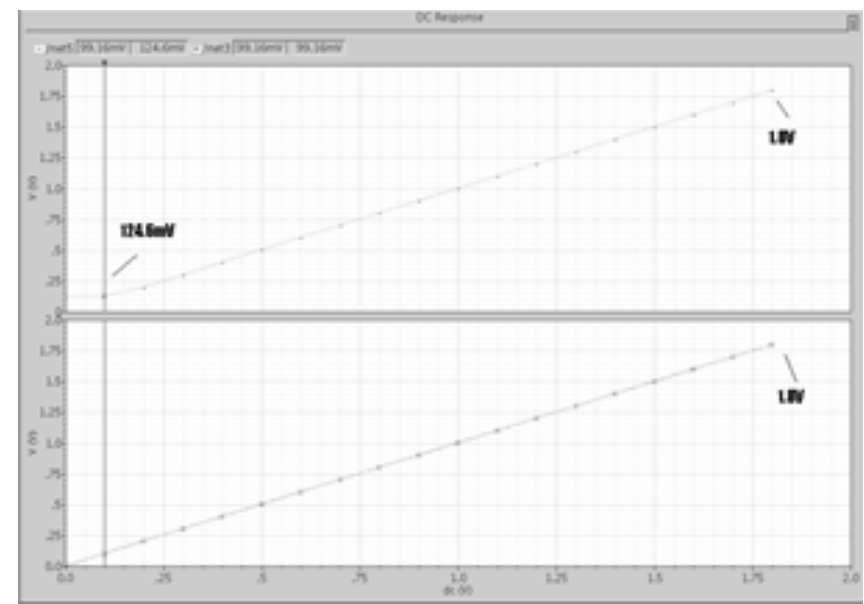

Figure 10-Output Swing of Differential Amplifier

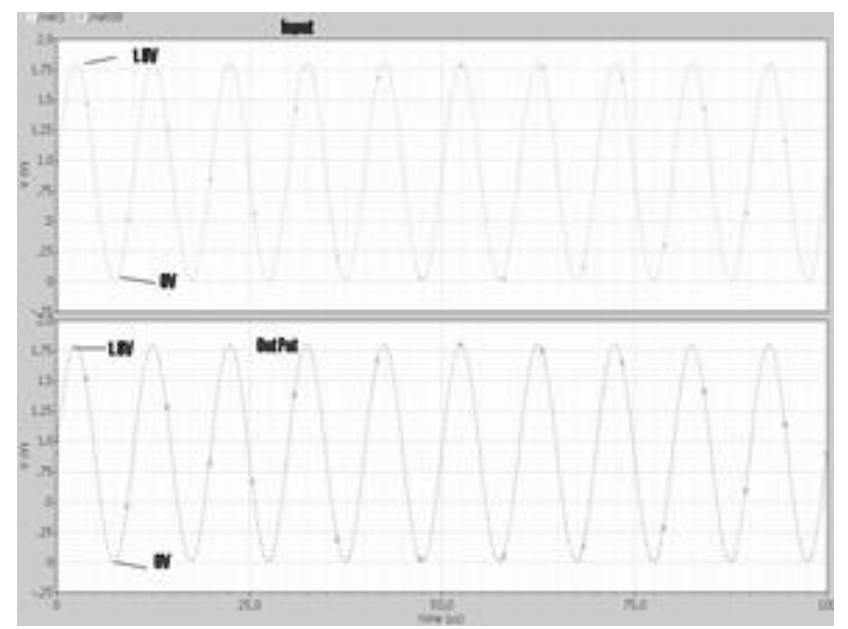

Figure 11-Rail to rail voltage swing considering sine wave input
Figure 11 presents the rail-to-rail voltage swing for a given sine wave input. The Vin is varied from 0 to $2 \mathrm{~V}$ and the output volta ge swing is measured be between $124.6 \mathrm{mV}$ to $1.8 \mathrm{~V}$. The dynamic range of the designed differential amplifier is 1.6754 and the ratio of dynamic range is $83.77 \%$. It is expected to be nearing $85 \%$; the DA design a chieves dynamic output voltage swing of $83.77 \%$. Figure 12 shows the configuration used to find the DC open loop gain of the system and the DC gain is the important parameter in the design ofDA circuit. The open loop ga in results are presented in Figure 13 and the open loop DC ga in is $103 \mathrm{~dB}$ which is higher than the desired requirement of $100 \mathrm{~dB}$.

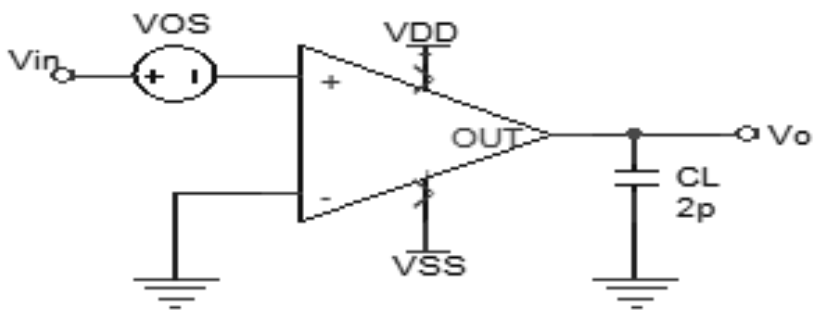

Figure 12-Configuration for DC open loop ga in

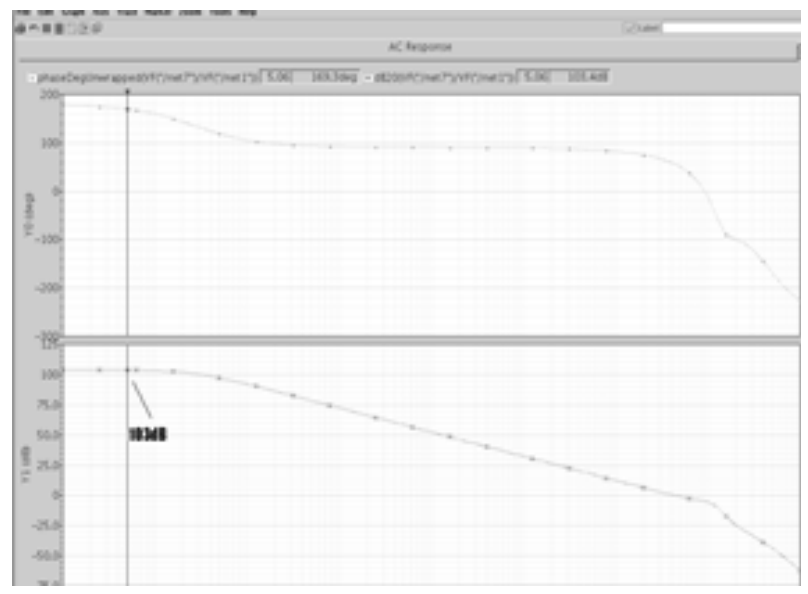

Figure 13-DC open loop ga in

Common Mode Rejection Ratio (CMRR) is measured considering the circuit configuration shown in Figure 14. As CMRR was critical parameter, necessary steps are required to be considered in design of transistor geometries to ensure to meet the transistor matching criteria. In this work, transistor geometries are fine tuned to meet the CMRR requirementsbased on simula tion results. The transistor geometries a re varied by a factor of $8 \%$ as compared with theoretical ca lculations. Further it is required to consider transistor matching during layout design that will provide the a ctual CMRR computations after back annotations. Figure 15 is the simulation result of CMRR for the designed DA circuit. 


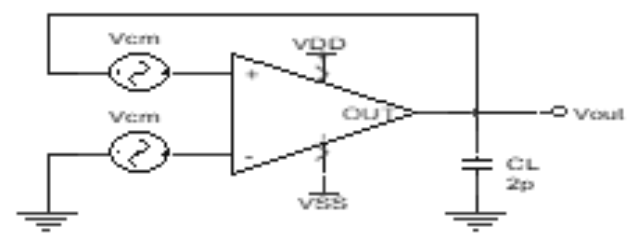

Figure 14-Configuration to measure CMRR

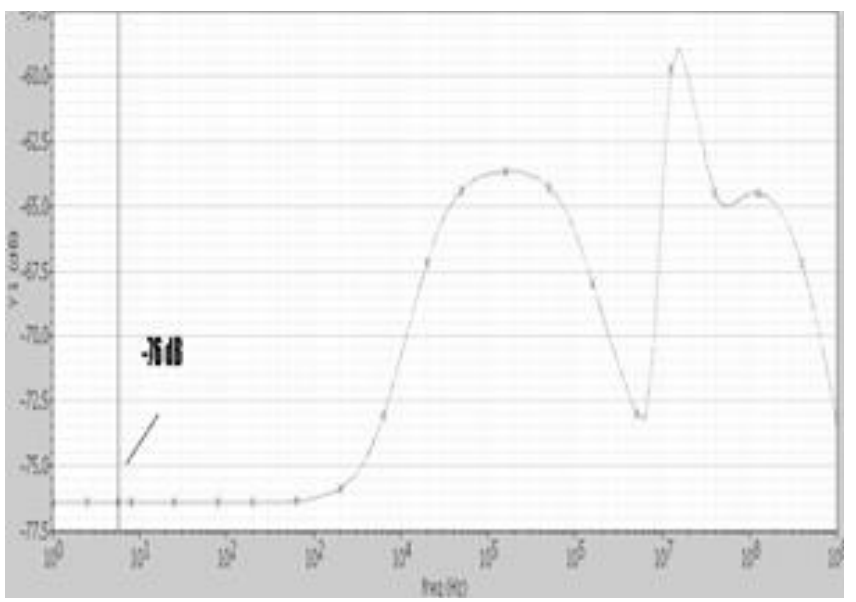

Figure 15-CMRR simulation results

The slew rate and settling time is mea sured for the DA circuit. The slew rate is found by connecting the DA in voltage follower configuration and input is set to a square wave. The slew rate is found to be $6 \mathrm{~V} / \mu \mathrm{sec}$ and settling time was $0.9 \mu \mathrm{sec}$ as shown in Figure 16.

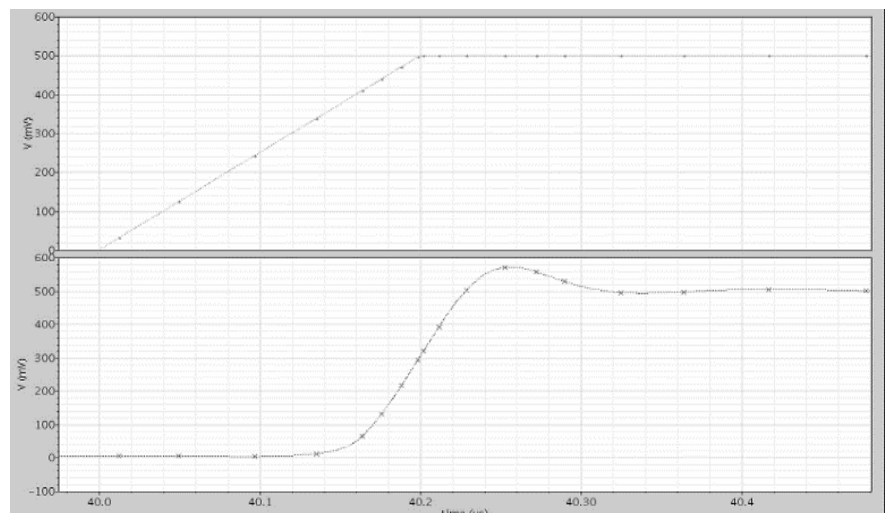

Figure 16-Slew rate results for DA

Power Supply Rejection Ratio (PSRR) is the capability for the DA to reject any noise being induced from the power supply. It is measured by connecting the DA as per the test configuration shown in Figure 17 and the corresponding simulation results are shown in Figure 18 from which the mea sured PSRR is $83 \mathrm{~dB}$.

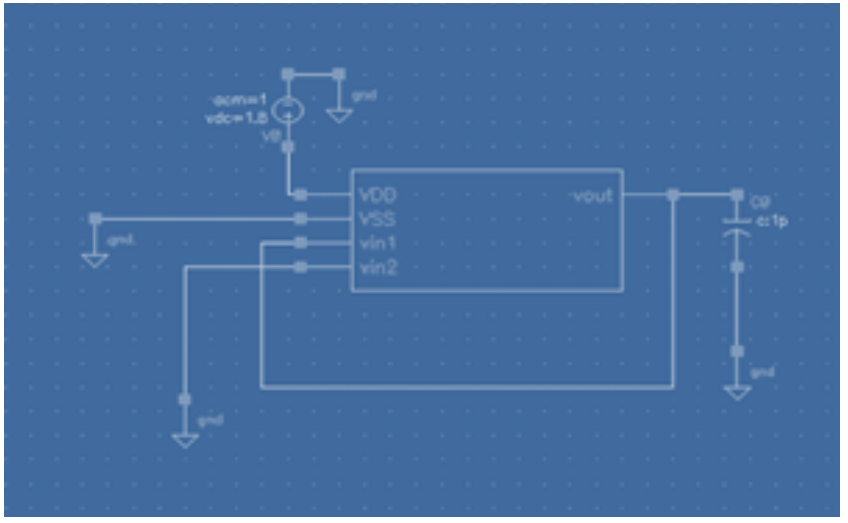

Figure 17-PSRR Configuration

Bin Wang et al. [18] have designed Asymmetric structure for power a mplifiers and have discussed a thorough methodology for analysis. Sami Laifa et al. [19] have designed PID Controllers for MIMO Systems. For implementation of this module differential amplifier designed in this paper can be adopted.

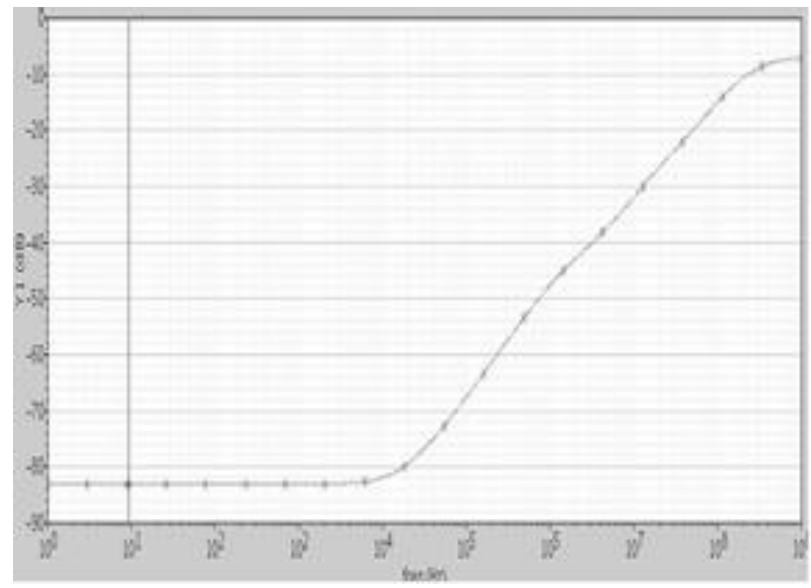

Figure 18-PSRR Simula tion results

The power supply rejection of the DA is simulated using ELDO tool. An external script used to measure the quantities. 1V AC added in order to verify the power supply rejection of DA. All the analog input is forced low-to-high to get the full-scale output. Then the power supply rejection is measured on the DA analog output. The resulting output waveform which is simulated across corners is shown in Figure 19. 


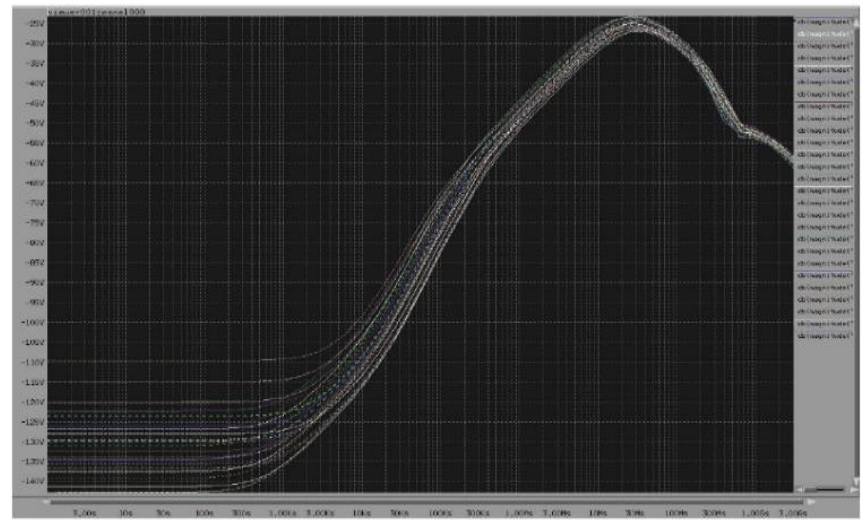

Fig. 19-Power supply rejection shown on DA output

The Power Supply Rejection Ratio (PSRR) is expected around $70 \mathrm{~dB}$ and it should not exceed $90 \mathrm{~dB}$. The a chieved PSRR is around $83 \mathrm{~dB}$. The power dissipation is $120 \mu \mathrm{W}$ with operating frequency of $10 \mathrm{GHz}$. It is required to reduce power dissipation and limit it to less than $50 \mu \mathrm{W}$; this is achieved with proper design of transistor geometries. Figure 20 presents the Transconductance Vs common mode voltage $\left(\mathrm{V}_{\mathrm{CM}}\right)$ variation for the designed DA. Input is varied and transconductance is ca lculated and corresponding gra phs generated. The variation in the transconductance is less than $0.71 \%$ demonstrating stability in the performances ofDA circuit.

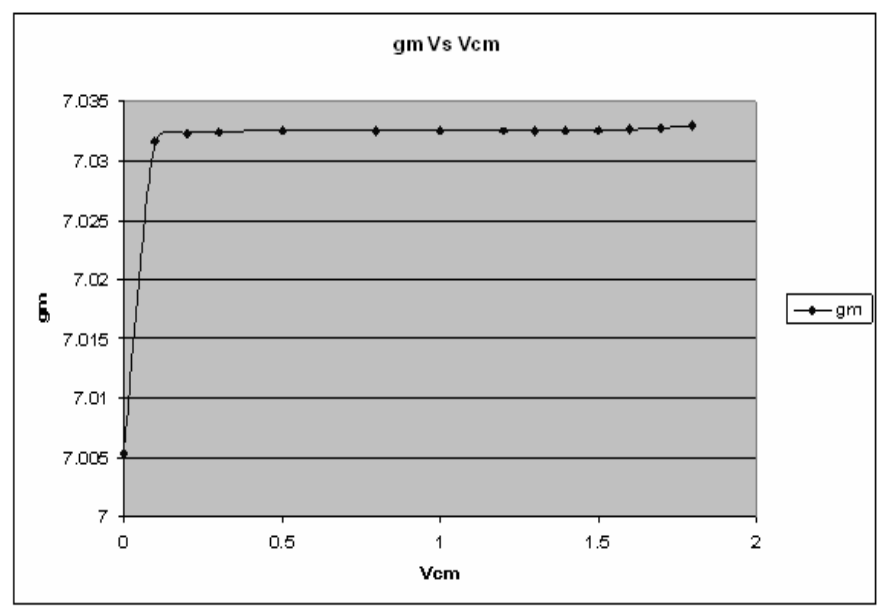

Figure 20-Constant Transconductance

The DA circuit is evaluated as comparator circuit. The simulation of the DA is carried out with a test case where a PWL signal is provided as the a nalog input to the DA. Power supply is set to $1.8 \mathrm{~V}$ and the input data is set at $80 \mathrm{MHz}$ is given as the clock. A delay (0.3ns) is a dded to the input to compensate the layout parasitic capacitance which will cause the input delay. The simulation results for the comparator operation are shown in the Figure 21.

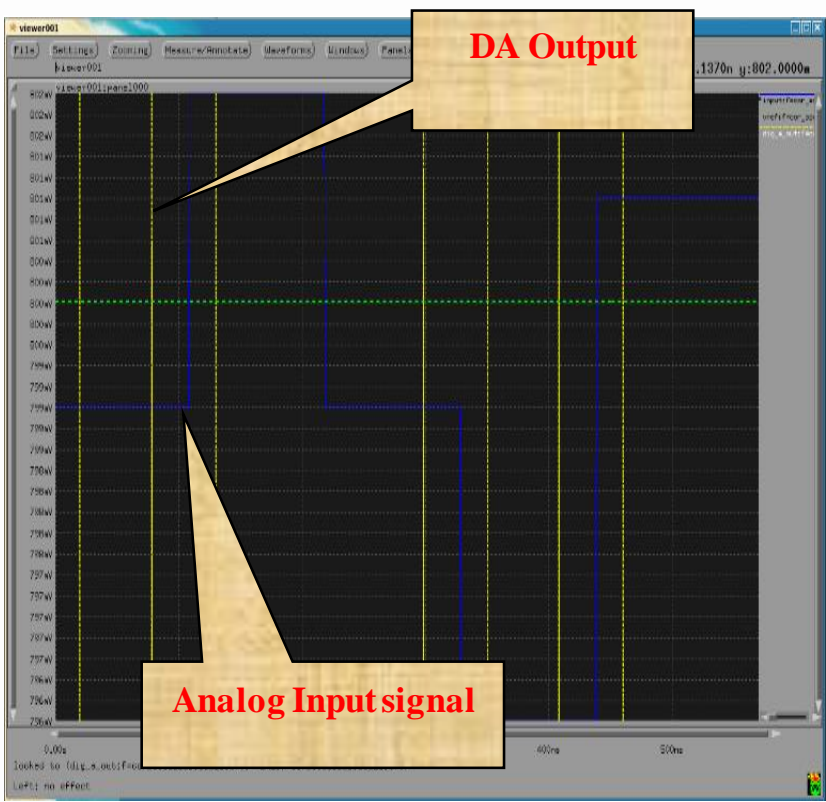

Fig. 21 Simulation results of DA

In this work Differential Amplifier Design Parameters are considered as VDD, DC Ga in, Power Supply Rejection Ratio (PSRR) Slew Rate, Power Dissipation and Common Mode Rejection Ratio (CMRR). The results obtained are tabulated in table 4 and the Percentage Improvement is ca lculated for a ll the parameters mentioned and compared.

Table 4-Comparison of DA Circuits

\begin{tabular}{|l|l|l|l|}
\hline $\begin{array}{l}\text { Differential } \\
\text { Amplifier } \\
\text { design } \\
\text { parameters }\end{array}$ & $\begin{array}{l}\text { Existing } \\
\text { literature } \\
\text { using 32nm } \\
\text { FINFET } \\
\text { technology [3] }\end{array}$ & $\begin{array}{l}\text { Present } \\
\text { work using } \\
\text { 22nm } \\
\text { FINFET } \\
\text { technology }\end{array}$ & $\begin{array}{l}\text { Percentage } \\
\text { improvement }\end{array}$ \\
\hline VDD & $1.8 \mathrm{~V}$ & $1 \mathrm{~V}$ & $80 \%$ \\
\hline DC Gain & $103 \mathrm{~dB}$ & $107 \mathrm{~dB}$ & $3.88 \%$ \\
\hline PSRR & $32.4 \mathrm{~dB}$ & $83 \mathrm{~dB}$ & $60.96 \%$ \\
\hline Slew Rate & $5 \mathrm{~V} / \mu \mathrm{S}$ & $6 \mathrm{~V} / \mu \mathrm{S}$ & $16.67 \%$ \\
\hline $\begin{array}{l}\text { Power } \\
\text { dissipation }\end{array}$ & $58 \mu \mathrm{W}$ & $120 \mu \mathrm{W}$ & $51.66 \%$ \\
\hline CMRR & $11.34 \mathrm{~dB}$ & $76 \mathrm{~dB}$ & $85.07 \%$ \\
\hline
\end{tabular}

The DA Circuit designed in this work has a ga in improvement of $3.88 \%$. This is the most significant for improving SNR. The PSRR has been improved by a factor of 50Decibels and is suitable for low voltage ADC's. The power dissipation of $120 \mu \mathrm{W}$ can be reduced further by reducing slew rate.

\section{CONCLUSION}

In this work a Differential amplifier circuit is designed that could be used for design high speed, low power ADC based on SAR logic using model parameters of high-k FINFET in $22 \mathrm{~nm}$ technology. The FINFET device parameters are considered from Predictive Technology Model (PTM). The VI Characteristics of FINFET is plotted considered at $22 \mathrm{~nm}$ 
technology with high-K dielectric. Output characteristics for varying width of $22 \mathrm{~nm}$ technology HIGH-k FINFET based inverter is evaluated. The gain and phase-margin of Differential Amplifier are identified to be greater than $107 \mathrm{~dB}$ and $63^{\circ}$ respectively. In this work a $1 \mathrm{~V}$ Supply is used as an input voltage, the Power dissipation is $50 \mu \mathrm{W}$ and the overall performance of Differential Amplifier circuit was improved by $4 \%$. The Power Supply Rejection Ratio (PSRR) is estimated to be around $83 \mathrm{~dB}$. Slew rate is $6 \mathrm{~V} / \mu \mathrm{s}$ and the ratio of dynamic range is $83.77 \%$. Also, the dynamic range of the differential a mplifier is found to be 1.6754 . The designed DA sub circuit is suitable for high speed ADC design operating at $100 \mathrm{MHz}$.

\section{ACKNOWLEDGEMENT}

The authors acknowledge the valuable suggestions given by Prof. Cyril Pra sanna Raj P. and Principal Dr. Subra manya K N of RV Engineering College, Bangalore for having permitted to use the Research facilities a vailable in esteemed college, also HOD of Electronics and Communication Department. We a cknowledge Cadence for providing us the License V6.7.11 for using the software for schematic capture and simulations under university program.

\section{References:}

[1] F. Ba lestra, S. Cristoloveanu, M. Benachir, J. Brini and T. Elewa, "Double-gate silicon-on-insulator transistor with volume inversion: A new device with greatly enhanced performance," in IEEE Electron Device Letters, vol. 8, no. 9, pp. 410-412, Sept. 1987, doi: 10.1109/EDL.1987.26677.

[2] Vahid Baghi Rahin, and Amir Baghi Rahin, "A Low-Voltage and Low-Power Two-Stage Operational Amplifier Using FinFET Transistors," vol. 3, no. 4,pp. 80-95, June 2016.

[3] A. Vikhe and S. Turkane, "Comparative performance analysis of single stage differential a mplifier at 32 nanometer regime," 2015 International Conference on Energy Systems and Applications, Pune, pp. 406-410, $30^{\text {th }}$ Oct- $1^{\text {st }}$ Nov 2015, doi: 10.1109/ICESA.2015.7503380.

[4] S. Hsieh, C. Ka o, and C. Hsieh, "A 0.5-V 12-bit SAR ADC Using Adaptive Time-Domain Comparator with Noise Optimization," in IEEE Journal of Solid-State Circuits, vol. 53, no. 10,pp. 2763-2771, Oct. 2018.

[5] Y. Chen, S. Tsukamoto, and T. Kuroda, "A 9b 100MS/s $1.46 \mathrm{~mW}$ SAR ADC in 65nm CMOS," In Proc. ASSCC, pp.145148, Nov. 2009.

[6] M. Yoshioka, K. Ishikawa, T. Takayama, and S. Tsukamoto, "A $10 \mathrm{~b} 50 \mathrm{MS} / \mathrm{s} 820 \mathrm{uW}$ SAR ADC with on-chip digital calibration," In ISSCC Dig. Tech Papers, pp. 384-385, Feb. 2010.

[7] H. Choi, Y. Kim, G. Ahn, and S. Lee, "A 1.2-V 12- b 120MS/s SHA-free dual-channel Nyquist ADC based on midcode calibration," IEEE Trans. Circuits Syst. I, vol. 56, no. 5, pp. 894-901, May 2009.

[8] Ta i-ji An, Young-Sea Cho, Jun-Sang Park, Gil-Cho Ahn, and Seung-Hoon Lee, "A Two-channel 10b 160 MS/s28 nm CMOS Asynchronous Pipelined-SAR ADC with Low Channel Mismatch," Journal of Semiconductor Technology and Science, vol. 17 , no. 5 , Oct. 2017.
[9] B. Murmann, "A/D converter trends: Power dissipation, scaling, and digitally assisted architectures," in Proc. IEEE Custom Integr. Circuits Conf. (CICC), pp. 105-112, Sep. 2008. [10] A. Baschirotto, V. Chironi, G. Cocciolo, S. D'Amico, M. De Matteis, and P. Delizia, "Low power analog design in scaled technologies," in Proc. Topical Workshop Electron. Particle Phys., Paris, France, pp. 103-110, Sep. 2009.

[11] C.-Y. Chen, J. Wu, J.-J. Hung, T. Li, W. Liu, and W.-T. Shih, "A 12-bit $3 \mathrm{GS} / \mathrm{s}$ pipeline ADC with $0.4 \mathrm{~mm}^{2}$ and $500 \mathrm{mw}$ in $40 \mathrm{~nm}$ digital CMOS," IEEE J. Solid-State Circuits, vol. 47, no. 4,pp. 1013-1021, Apr. 2012.

[12] Tosaka, Y., Suzuki, K., Horie, H., \& Sugii, T., Scalingparameter-dependent model for subthreshold swing $S$ in double-gate SOI MOSFET's. IEEE Electron Device Letters, vol. 15, no. 11,pp. 466-468, Nov 1994.

[13] Chenming HU, Tsu-Jae King-Liu and Jeffrey Bokor, "FinFET-A Self Aligned Double -Gate MOSFET Scalable to 20nm," IEEE Transactions on Electronic Devices, vol. 47 no.12, pp. 2320-2325, Dec 2000.

[14] Colinge, JP., and Editors. FinFET and Other Multi-Gate Transistors. pp 1-13 Springer, 2008.

[15] A. Amara, O. Rozeau, and Editors, "Pla nar Double-Gate Transistor: From Technology to Circuit," Springer, pp. 1-20, 2009.

[16] Moon Seok Kim, Huichu Liu, Xueqing Li, Suman Datta, and Vijaykrishnan Narayanan, "A Steep-Slope Tunnel FET Ba sed Sar Analog-To-Digital Converter," IEEE Transactions on Electron Devices, vol. 61, no. 11,pp. 3661-3667, Nov 2014.

[17] P. E. Allen, and D. R. Holberg, " CMOS Analog Circuit Design," $2^{\text {nd }}$ Ed. New York: Oxford University Press, 2002.

[18] Bin Wang, Jiang Teng, Xin Zhang, Dong Su and Wei Luo, "High-Efficiency Doherty Power Amplifier Based on an Asymmetric Structure,"Engineering World, vol. 2, pp. 264-269, 2020.

[19] Sa mi Laifa, Badreddine Boudjehem, and Djalil Boudjehem, "Analytical Design of Multi-loop Fractional IMC-PID-Filter Controllers for MIMO System Using Equivalent NIOPDT Models," WSEAS Transactions on Systems and Control, ISSN / E-ISSN: 1991-8763 / 2224-2856, vol. 15, Art. \#40, pp. 383393,2020.

\section{Creative Commons Attribution License 4.0 (Attribution 4.0 International, CC BY 4.0)}

This article is published under the terms of the Creative Commons Attribution License 4.0 https://creativecommons.org/licenses/by/4.0/deed.en_US 